\title{
Rethinking the Boundary Conditions in the Particle-in-a-Box Mind Experiment
}

Jixin Chen

Department of Chemistry and Biochemistry, Ohio University, Athens Ohio 45701

chenj@ohio.edu

\begin{abstract}
In this manuscript, I speculated that the energy density distributions along space and time in a quantum system are uniform. Thus, the complementary energy contributions are added to the classical solutions of the 1D particle in a box problem, making the energy density a complex distribution function over space and time. Then the concept is extended to the free rotation problem with a Hamiltonian slightly different than the classical Schrödinger equation. The picturized energy distribution functions and associated time evolution are described in movies for comparison between example classical wave functions and the energy density functions. The wave functions for the hydrogen atom are then guessed based on the historical solutions.
\end{abstract}

\begin{tabular}{|l|l|l|l|l|}
\hline TOC: Guessed energy density function shapes (absolute) of carbon-like and oxygen-like atoms. \\
\hline-1
\end{tabular}

I had trouble explaining the 1D particle-in-a-box mind experiment to my students when I was teaching an undergraduate physical chemistry class at Ohio University. The experiment states that there is a space of zero potential energy sandwiched between two walls with infinite potentials and a particle say an electron, is put inside the potential well. ${ }^{1}$ The wavefunction of this particle must satisfy the continuous assumption as a well-behaved curve thus for the ground state, the probabilities of seeing this particle approaching zero near the walls and maximized at the center of the well. The excited-state wavefunctions have maximums and nodes yielding a probability density function over the space shown in Fig. 1A. A student asked during my lecture, why was the probability near the walls zero? I suddenly lost the reason to answer this question due to my limited knowledge of quantum mechanics. So, I said that the wavefunction "should" be continuous and made up an explanation by analogy it to the vibration of a guitar string that the two ends do not move. 


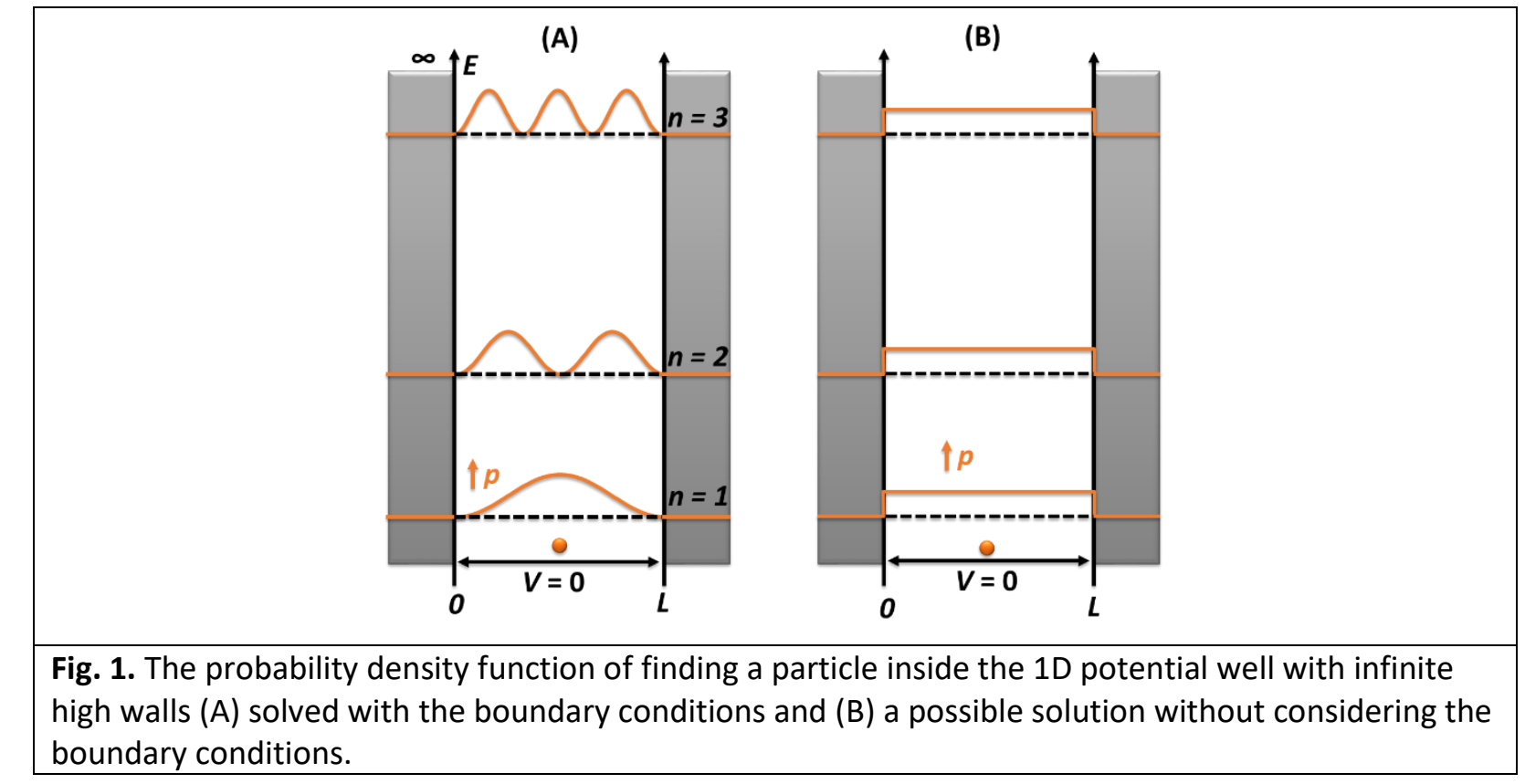

But in fact, I was not sure about my answer. The problem lies in that for a real-world experiment, we will never have a potential well to have walls with infinite potentials that break the continuity of space and time along the $x$-axis. Thus, it makes sense to have a continuous wave function along with the whole space in the real world. In this mind experiment, we break the continuity of space and time but still expect that the wave function is continuous. This expectation seems not physical.

What if we give up the continuity requirement of the wave function for this special case and instead assume uniform energy density over space and time? Different boundary conditions suddenly open, specifically, the space and time outside of the wall still give a wavefunction of zero but binary values at the wall. If these breaking points are allowed to compensate for our irrational assumption of the existence of potential walls with infinite values, we should expect a probability density function shown in Fig. 1B. If we want, we can even assume that a single Planck length from a wall is needed for the wavefunction to jump from a value to zero to enforce its continuity.

So I give it a try to solve the time-dependent Schrödinger equation with the no-boundary assumption:

$$
i \hbar \frac{\partial}{\partial t} \psi(x, t)=-\frac{\hbar^{2}}{2 m} \frac{\partial^{2}}{\partial x^{2}} \psi(x, t)+V(x) \psi(x, t)
$$

where the imaginary number $i=\sqrt{-1}, \hbar$ is the reduced Planck constant, $m$ is the mass of the particle, $\Psi(x, t)$ is the wave function, and $V(x)$ is the potential profile. Without the boundary restriction, all wavefunctions of a free particle in space satisfy this equation:

$$
\psi(x, t)=\frac{1}{L} e^{ \pm i u x \pm i \omega t}
$$

where $u$ and $\omega$ are both arbitrary values.

To meet the experimental observation that energy level is quantized, symmetry argument is required to restrict the values the same as the original arguments in history, e.g. those among Planck, Einstein, Bohr, 
de Broglie, Born, Dirac, Heisenberg, Pauli, Schrödinger, and ... Let's assume that the left and right of the well have an identical probability in any given time to have the same amplitude of momentum, i.e. at a resonant state. This argument shrinks the solutions to $u_{n}=\frac{n \pi}{L}$ and $\omega_{n}=\frac{n^{2} \pi^{2} \hbar}{2 m L^{2}}$ where $n=1,2,3, \ldots$ is a positive integer. A comparison among the wavefunctions solved with the boundary and without the boundary conditions is shown in Fig. 2.

The energy solutions converge to the classical solutions in the textbooks. However, we see a very different shape of the wavefunctions $\psi(x, t)$ (Fig. 2) and probability density functions $|\psi(x, t)|^{2}$ (Fig. 1). There is an interconversion between the real part and the imaginary part of the wavefunction which maintains the modulus of the wavefunction constant across the well (Fig. 1B), i.e. it is now equally possible to observe a particle at anywhere of the well. I assume that the real part can be the electric field and the imaginary part can be the magnetic field of the particle in the well that resonant and maintain the energy of the particle for a measurable period (Fig. 2B).

At any given time, the sum of the "momentum" in the magnetic field is zero, and/or the sum of the "momentum" of the electric field is zero. I guess any non-resonant frequency decays to the energy levels by radiating electromagnetic waves when the product of the two sums is not zero that breaks the conservation law. After all, the whole energy equation is Maxwell's electromagnetic wave function. Since the wavefunctions belong to a subset of the free-space matter-waves, the Heisenberg uncertainty principle still holds and the two sets of the solutions are entangled together in the momentum space.

(A)

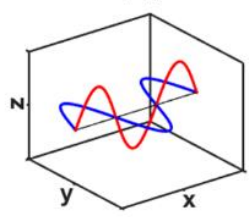

$\Psi_{1}(x, t) n=3$

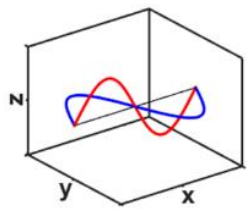

$\Psi_{1}(x, t) n=2$

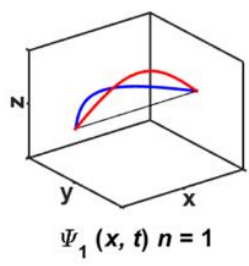

(B)

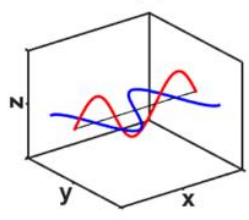

$\Psi_{2}(x, t) n=3$

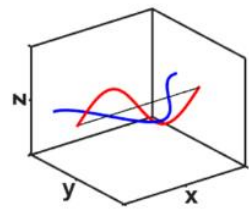

$\Psi_{2}(x, t) n=2$

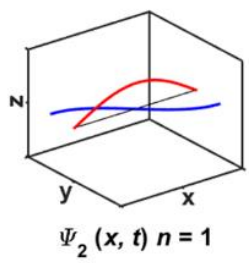

Fig. 2. A few examples $(n=1,2,3)$ of the real ( $z$-axis) and imaginary ( $y$-axis) parts of the wavefunction at time zero of the solutions with (A) zero probability densities, and (B) equal probability densities at the boundaries vs anywhere inside the classical 1D particle-in-a-box well ( $x$-axis from 0 to $L$ ). The probability density function is the square modulus of the two curves where the left yields a probability density curve with nodes (Fig. 1A) and the right yields a flat line along the $x$-axis (Fig. 1B) inside the well. See attached a movie created using MATLAB and a video converting code. ${ }^{2}$ Time evolution is shown in SI video 1. 
We can add back the missing energy in the original Schrödinger equation to make the energy density equals everywhere in space and time. We can also modify the stationary differential equation to

$$
-\frac{\hbar^{2}}{2 m}\left(\frac{\partial \psi(x, t)}{\partial x}\right)^{2}+V(x) \psi(x, t)=E \psi(x, t)
$$

We can still use the original equation to quantize the energy levels. Put Equation 2 into Equation 3, we got the stationary-state energy to be:

$$
E_{n}=\frac{\hbar^{2} k^{2}}{2 m L^{2}} e^{ \pm i k x}
$$

Where $k_{n}=\frac{n \pi}{L}$. Equation 4 says that the energy density is evenly distributed in the 1D well. However, there is an interconversion between the real energy and the imaginary energy that follows a complementary symmetry and obeys the energy conservation law.

For the rigid free rotator problem, the Hamiltonian becomes,

$$
-\frac{\hbar^{2}}{2 m R^{2}}\left[\left(\frac{\partial \psi(\theta, \varphi, t)}{\partial \theta}\right)^{2}+\left(\frac{\partial \psi(\theta, \varphi, t)}{\partial \varphi}\right)^{2}\right]+V(\theta, \varphi, t) \psi(\theta, \varphi, t)=E \psi(\theta, \varphi, t)
$$

Where $R$ is the radius of the rotator, $\theta$ and $\varphi$ are the rotation angles, and $V=0$. Based on the solutions on Equation 1, I guess a set of stationary wave functions:

$$
\psi_{j, k}(\theta, \varphi)=e^{ \pm i j \theta} e^{ \pm i k \varphi}
$$

Where $j$ and $k=0,1,2,3 \ldots$ And

$$
E_{j, k}=\frac{\left(j^{2}+k^{2}\right) \hbar^{2}}{2 m R^{2}} e^{ \pm i j \theta} e^{ \pm i k \varphi}
$$

There are many problems with these solutions. Among them, the energy gaps between energy levels are different than that of the classical solutions, e.g. from classically $1,1,4,6,8,10 \ldots$ to $1,1,3,3,5,5, \ldots$ times $\hbar /\left(2 m R^{2}\right)$. A comparison between shapes of the classical solutions and the new solutions are shown in Fig. 3, and Fig. 4 with the time evolution shown in the SI video 2 and video $\mathbf{3}$ respectively. Larger quantum numbers give more structures with an example of $j= \pm 2$ and $k= \pm 2$ shown in video 4 . 

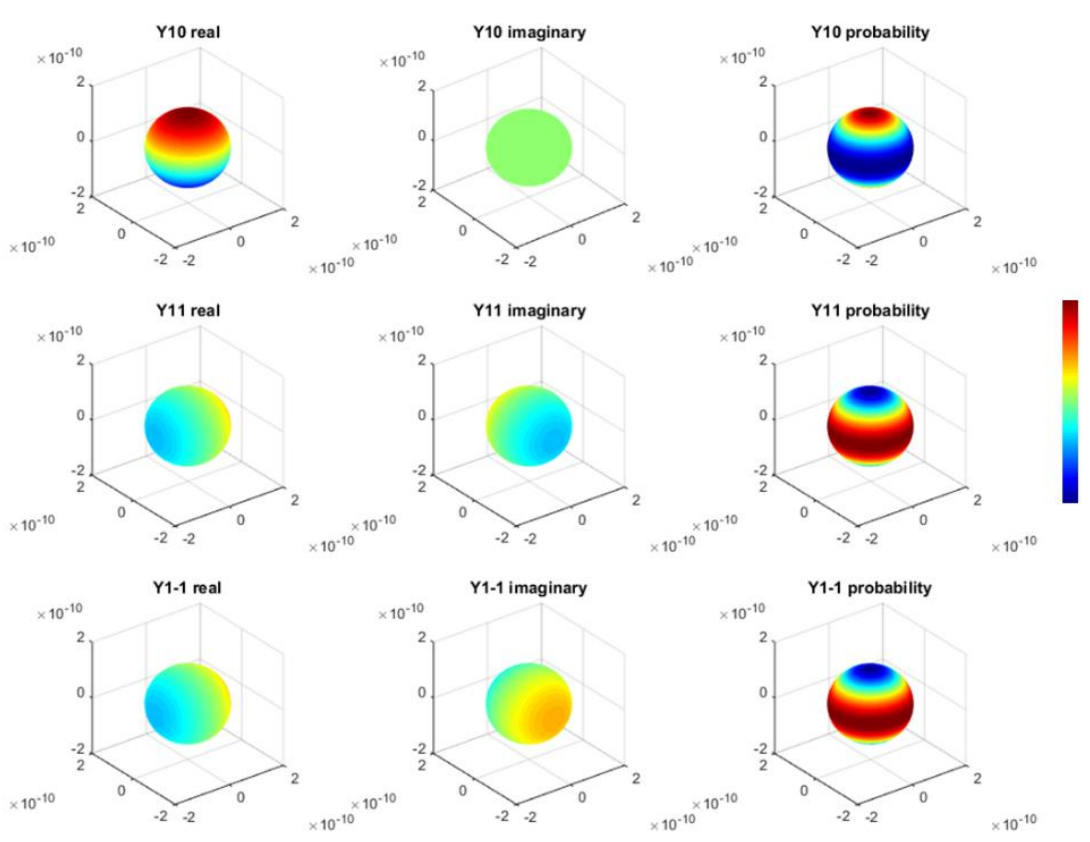

Y1-1 probability

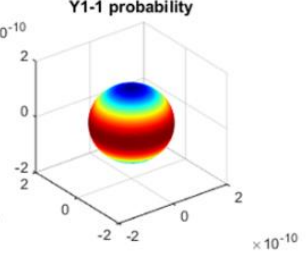

Fig. 3. Example classical solutions of rigid rotator free rotation wave functions with angular quantum number 1 at time zero.
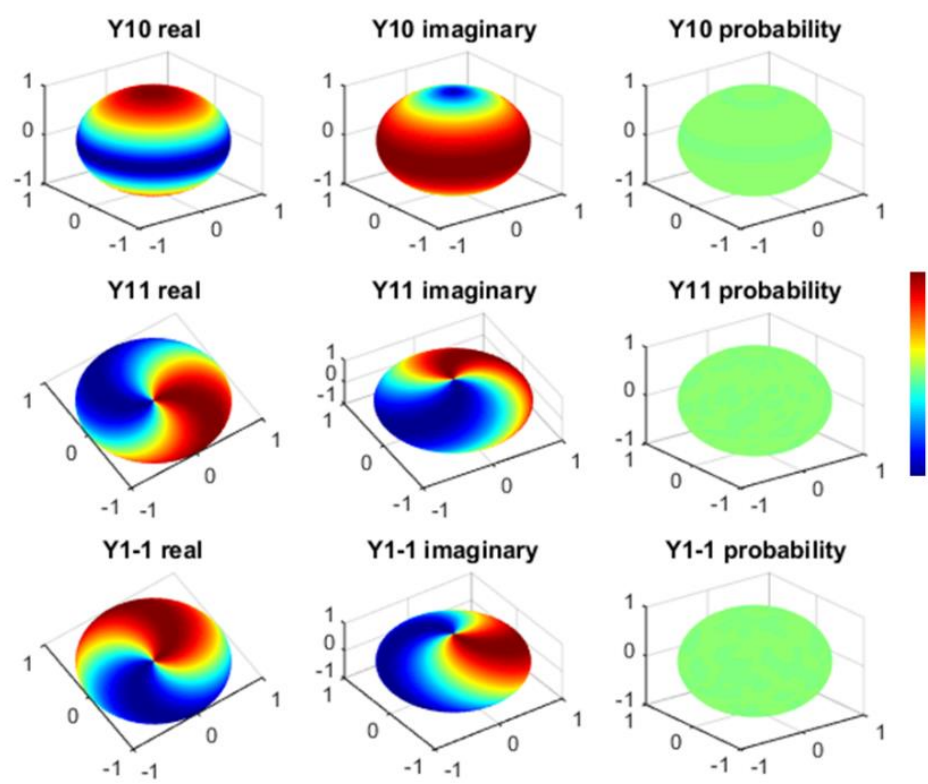

Y1-1 probability

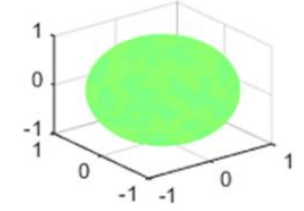

Fig. 4. Example solutions of rigid rotator free rotation new energy density function with angular quantum numbers $(1,0),(1,1)$, and $(1,-1)$.

Another problem with these solutions is we lose the node structure in the new energy density functions in contrast to the node structure in the probability density functions. I speculate that the real part of the 
energy density function is electric field energy, and the imaginary part of the energy density function is magnetic field energy or vice versa. Again, these equations resemble Maxwell's equation of electromagnetic waves of massless photons. Thus, we still have nodes for the electric part and the magnetic part of the wave function that are orthogonal to each other, which can be tested by measuring the nodes with electric and magnetic methods. A problem is over time the real part and the imaginary part both evolve into chiral structures for these solutions when both $j$ and $k$ are none zero, whose physical meaning is unclear.

Other than these problems, I hope you agree that rethinking the boundary conditions in the classicalquantum mechanical problems are useful practices for researchers and students. The main idea is that energy is equally distributed in space. The mathematical equations work consistently with the classical equations for the idealized mind experiments with a lot of "spookiness" vanishes in the new model.

Based on the above theory, I then give it a random guess on the new wavefunctions of atoms. For the hydrogen atom, based on the historically established solutions, I guess an un-normalized solution as

$$
\psi_{n, l, m}(r, \theta, \varphi, t)=H_{n}(r) e^{ \pm \frac{i r}{n a_{0}}} e^{ \pm i l \theta} e^{ \pm i m \varphi} e^{ \pm i \omega t}
$$

Where $H_{n}(r)$ is the Hermite polynomial, and $a_{0}$ is the first Bohr radius.

This wavefunction is very easy to add up, showing consistent shapes of the energy functions for atoms such as carbon and oxygen (Fig. 5).

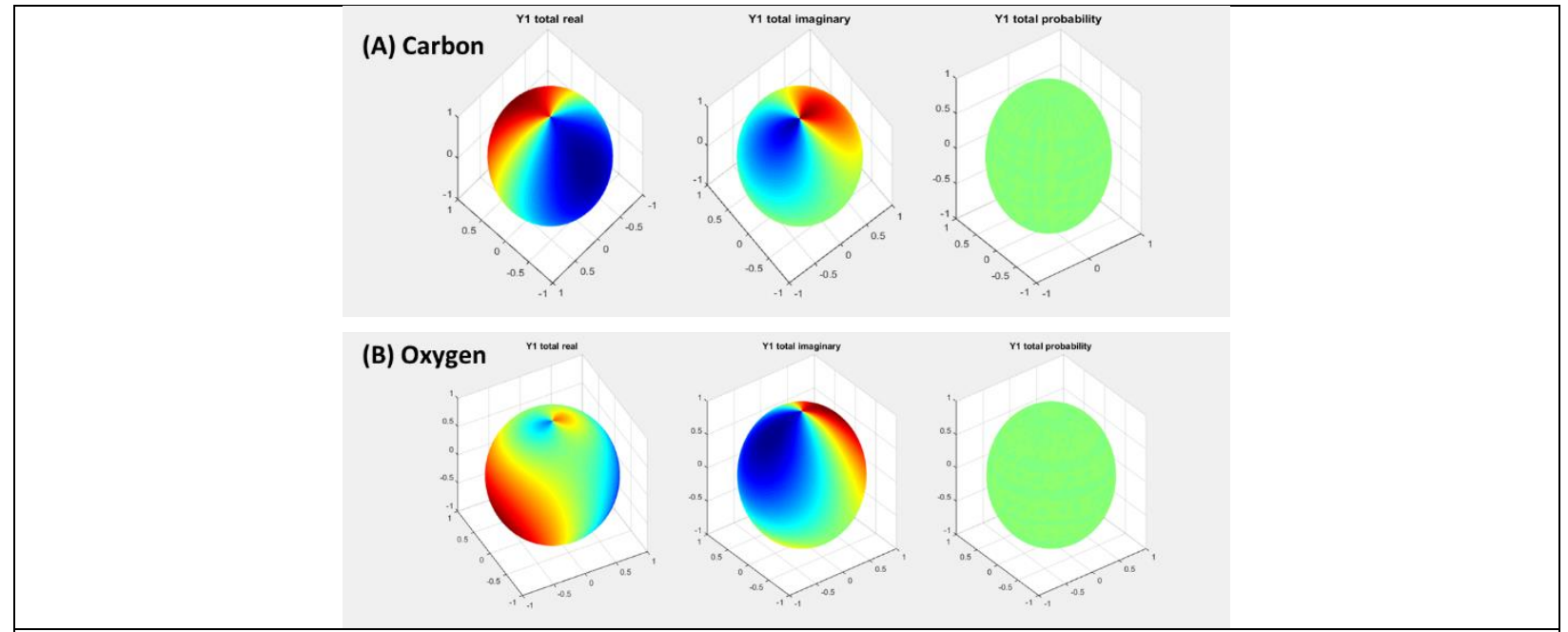

Fig. 5. Sum up the energy function to get bald guesses of the shape of $(A)$ carbon with $I=0,-1$ and $m=$ \pm 1 , (B) oxygen with $I=1$ and $m= \pm 1$. Explains well the tetrahedral structure in carbon and the water structure.

\section{Supporting information}

Videos and MATLAB source codes are attached to the supporting information. 


\section{References}

Note: Too many giants have contributed to this field. Historical named references are not listed again here.

1. Raff LM. Principles of Physical Chemistry. Prentice Hall Upper Saddle River, NJ; 2001.

2. Lindner M. AVI to GIF convertor. Published online 2021.

https://www.mathworks.com/matlabcentral/fileexchange/28772-avi-to-gif-convertor 